\title{
A Integração Ensino-Serviço como Estratégia na Formação Profissional para o SUS
}

\section{Integration between Learning and Health Services as a Strategy for Professional Training in the Unified Health System}

\author{
Adolfo Pizzinato III \\ Andréia da Silva Gustavo \\ Beatriz Regina Lara dos Santos ${ }^{I}$ \\ Beatriz Sebben Ojeda \\ Eliana Ferreira ${ }^{I I I}$ \\ Flávia Valladão Thiesen ${ }^{I V}$ \\ Marion Creutzberg ${ }^{l}$ \\ Marisa Altamirano \\ Oscar Paniz ${ }^{V I}$ \\ Valéria Lamb Corbellini ${ }^{\text {VIII }}$
}

PALAVRAS-CHAVE:

- Atenção Primária à Saúde;

- Integração Docente-Assistencial;

- Educação;

- Sistema Único de Saúde.

\section{KEYWORDS:}

- Primary Health Care;

- Teaching Care Integration;

- Education;

- Unified Health System.
Aprovado em: $30 / 11 / 2010$
I Pontifícia Universidade Católica do Rio Grande do Sul, Porto Alegre, RS, Brasil.

"I Gerente do Distrito Leste/Nordeste do Município de Porto Alegre, Porto Alegre, RS, Brasil.

III Professora Titular da Faculdade de Farmácia.

IV Secretaria Municipal de Saúde de Porto Alegre, Porto Alegre, RS, Brasil.

${ }^{v}$ Conselho Municipal de Saúde de Porto Alegre.

\section{RESUMO}

Trata-se de um relato de experiência relativo às estratégias e ações adotadas para a integração ensino-serviço, com a finalidade de contribuir no processo de formação profissional na área da saúde, em consonância com as diretrizes e princípios do Sistema Único de Saúde (SUS). O trabalho aborda as descobertas, os avanços, as potencialidades, as dificuldades e os pontos críticos encontrados, assim como as experiências bem-sucedidas e a sustentabilidade vivenciada no contexto da Atenção Primária à Saúde com os programas Pró-Saúde e PET-Saúde.

\section{ABSTRACT}

This is a case report on an experience with strategies and actions for the integration of learning and health services, with the aim of contributing to professional training in health, in keeping with the guidelines and principles of the Unified National Health System (SUS). The study addresses the discoveries, advances, potentialities, difficulties, and critical points, as well as successful experiences and sustainability in the context of Primary Healthcare with the Pró-Saúde and PET-Saúde programs. 


\section{INTRODUÇÃO}

A parceria entre a Pontifícia Universidade Católica do Rio Grande do Sul (PUC-RS) e a Secretaria Municipal de Saúde (SMS) de Porto Alegre existe desde a década de 1970, com as Faculdades de Medicina, Psicologia e Serviço Social e, mais recentemente, a partir da década de 1990, com a inclusão de novos cursos da área da saúde, como Farmácia, Enfermagem, Fisioterapia e Nutrição.

Com a participação dessas áreas nos programas Promed, Pró-Saúde I e II e PET-Saúde, diversos projetos relacionados à inserção dos alunos nos serviços de saúde vêm sendo desenvolvidos, estreitando as relações e facilitando a integração entre docentes, acadêmicos, trabalhadores e gestores de saúde do município.

Assim, este artigo é um relato de experiência que aborda as estratégias e ações adotadas para a integração ensino-serviço, com a finalidade de facilitar o processo de formação profissional na área da saúde, em consonância com as diretrizes e princípios do Sistema Único de Saúde (SUS). Também aponta as descobertas, os avanços, as potencialidades, as dificuldades e os pontos críticos encontrados, assim como relata as experiências bem-sucedidas e a sustentabilidade vivenciadas no processo de formação profissional, facilitado pela integração ensino-serviço no contexto da Atenção Primária à Saúde.

Entende-se por integração ensino-serviço o trabalho coletivo pactuado, articulado e integrado de estudantes e professores dos cursos de formação na área da saúde com trabalhadores que compõem as equipes dos serviços de saúde, incluindo-se aí os gestores, cuja finalidade é a qualidade de atenção à saúde individual e coletiva, a excelência da formação profissional e o desenvolvimento/satisfação dos trabalhadores dos serviços. ${ }^{1}$

A cidade de Porto Alegre está dividida em oito Gerências Distritais, de acordo com o Orçamento Participativo do Município. Atualmente, a base territorial e populacional definidas para a prática dos cursos da área da saúde é constituída pelos Distritos de Saúde Leste e Nordeste, que pertencem à Gerência Distrital Leste/Nordeste, responsável pela gestão dos serviços de saúde dessas regiões do município.

O Distrito de Saúde Leste é constituído por 118.969 habitantes, distribuídos em 168 setores censitários. A proporção da população entre 0 e 4 anos de idade é de 8,75\%; de 60 anos é de 10,04\%; e 93,11\% é a proporção de alfabetizados. Assim, o distrito apresenta indicadores sociodemográficos próximos ao perfil médio do município; porém, ao se analisar sua composição por setores censitários, identificam-se contrastes muito marcantes. ${ }^{2}$
A taxa de mortalidade infantil global por 1.000 nascidos vivos é de 22,60. A proporção de óbitos de adolescentes entre 10 e 19 anos idade por violência, em relação a todos os óbitos, é de $5 \%$, e de óbitos de mulheres em idade fértil é de $8,0 \%$. As principais causas de morte são: agressão por arma de fogo, infarto do miocárdio e doenças associadas ao HIV. ${ }^{2}$

O Distrito Leste possui 14 Unidades Básicas de Saúde, compostas por um Centro de Saúde, três Unidades Básicas, quatro Unidades do Serviço de Saúde Comunitária do Grupo Hospitalar Conceição e seis Unidades de Estratégia Saúde da Família. Trabalhadores dos serviços de saúde do Distrito Leste, quando questionados sobre a qualidade dos serviços, apontam, dentre outros aspectos, preocupações com o acolhimento dos usuários, sobrecarga de trabalho, impotência diante da demanda, relações conflituosas e limitação de recursos. Tais pontos são passíveis de intervenção conjunta da rede de serviços de saúde, rede social e Universidade. ${ }^{3}$

O Distrito Sanitário Nordeste, por sua vez, é constituído de 55.081 habitantes, distribuídos em 66 setores censitários. A proporção da população entre 0 e 4 anos idade é de 11,37\%; de 60 anos é de 6,30\%; e 93,11\% é a proporção de alfabetizados. A taxa de mortalidade infantil global por 1.000 nascidos vivos é de 13,94\%. A proporção de óbitos de adolescentes entre 10 e 19 anos de idade por violência, em relação a todos os óbitos, é de 5,2\%, e de óbitos de mulheres em idade fértil é de $11,7 \%{ }^{3}$

Existe uma diferença importante e preocupante quanto à taxa de mortalidade infantil do Distrito Leste em relação ao Nordeste, especialmente considerando que essa taxa é um bom indicador para avaliar a condição de saúde da população de uma forma geral. No primeiro distrito, tem-se uma taxa de mortalidade infantil caracterizada como média e, no segundo distrito, como baixa. ${ }^{4}$ Buscar as causas de tal disparidade, bem como estratégias para sua redução, é crucial para a atenção à saúde da população dessas localidades. Há necessidade de reavaliar as causas da mortalidade das mulheres em idade fértil, pois se os óbitos forem ligados à gravidez, ao aborto, ao parto e ao puerpério eles podem, em sua grande maioria, ser evitados. ${ }^{5}$

Em 2006, o curso de graduação em Enfermagem da PUC-RS foi contemplado no edital do Pró-Saúde I, dos Ministérios da Educação e da Saúde, mobilizando uma mudança curricular ocorrida ainda naquele ano. No período de implantação do Pró-Saúde I, destaca-se o esforço para que as ações desenvolvidas tivessem caráter interdisciplinar, envolvendo ações conjuntas de ensino/pesquisa/extensão da Enfermagem com outras áreas da saúde, a exemplo da Nutrição e da Fisioterapia, a fim de potencializar os recursos previstos no programa. Tais ações foram decorrentes de discussões que se ampliaram 
com a parceria permanente da SMS e do Conselho Municipal de Saúde (CMS). Entre as estratégias para fortalecer tais parcerias, incluiu-se a realização de reuniões e contatos com a SMS e a Coordenadoria Geral da Rede de Atenção Básica (CGRABS) de Saúde do Município de Porto Alegre e suas respectivas equipes assessoras.

Os projetos propostos pelo Pró-Saúde I, aprovados pelo Ministério da Saúde, foram discutidos e adequados pela Comissão Gestora Local do Pró-Saúde/Enfermagem PUC-RS, em conjunto com trabalhadores de saúde e docentes, a fim de atender às demandas assistenciais e pedagógicas dos serviços de saúde e da formação dos futuros profissionais dessa área.

Uma das estratégias utilizadas foi os seminários itinerantes, realizados com as equipes das Unidades Básicas de Saúde (UBS) e de Saúde da Família (ESF), com o objetivo de apresentar o programa e seus objetivos, promovendo discussões iniciais com a equipe sobre o desenvolvimento das ações de saúde. No total, realizaram-se 16 seminários, que contaram com a participação de 198 trabalhadores da saúde.

Essa estratégia buscou, entre outras coisas, potencializar a construção coletiva, aproximando a academia com os trabalhadores e tornando todos copartícipes das experiências inovadoras no processo de ensino-aprendizagem, na formação de profissionais de saúde. $^{6}$

Simultaneamente ao desenvolvimento do Pró-Saúde I, a PUC-RS empreendeu esforços para a criação do seu Programa de Residência Multiprofissional em Saúde (Premus/PUC-RS), integrando-se ao movimento promovido pelos Ministérios da Saúde e da Educação com vistas à formação de profissionais para atender aos princípios do SUS. O Premus/PUC-RS recebeu, em 2006, parecer técnico favorável da Secretaria de Gestão do Trabalho e da Educação/MS. O processo seletivo dos residentes contou com a inscrição de 354 profissionais vindos da Região Metropolitana, do interior do Rio Grande do Sul e de estados vizinhos.

O programa se desenvolveu no período de 2007 a 2009, formando 42 profissionais nas áreas de Enfermagem, Farmácia, Fisioterapia, Nutrição, Odontologia, Psicologia e Serviço Social. Nesse programa foram enfatizadas três áreas: Saúde da Criança; Saúde do Adulto; e Atenção à Saúde. Áreas essas ligadas a nove campos de saber e prática: Saúde da Criança e Adolescente; Saúde da Mulher; Saúde do Idoso; Reabilitação Cardiopulmonar; Nefrologia; Intensivismo Adulto; Intensivismo Pediátrico; Saúde Bucal; e Saúde Mental. Dada a complexidade do programa, foi necessária a participação de múltiplos atores em diferentes instâncias da Universidade e dos serviços de saúde para garantir seu propósito interdisciplinar. O processo de implantação do Premus/PUC-RS proporcionou aos profissionais envolvidos a possibilidade de produção de novas práticas de cuidado à saúde com a implantação e qualificação de modelos assistenciais que contemplam a interdisciplinaridade, a integralidade e a humanização nos diferentes campos de saber e de prática.

A partir das vivências resultadas da 1ª̣ edição do Premus, foram evidenciadas a motivação e a expectativa dos serviços e dos profissionais de saúde quanto à continuidade do programa. Em agosto de 2009, teve início a sua $2^{\underline{a}}$ edição, agora como Programa de Residência Multiprofissional em Saúde da Família e Comunidade, contando com a participação de 19 residentes de sete áreas (Enfermagem, Fisioterapia, Nutrição, Odontologia, Serviço Social, Psicologia e Farmácia).

Em 2010, a PUC-RS foi contemplada com um novo Programa de Residência Multiprofissional e em Área Profissional da Saúde, no Hospital São Lucas da PUC-RS - Premus/HSL 2010, que ocorrerá simultaneamente ao programa já em andamento, alinhando-se às prioridades políticas de formação de recursos humanos do Ministério da Saúde. O Premus/HSL 2010 oferece 12 vagas distribuídas entre as áreas nele já existentes, acrescida a da Educação Física, contemplando, portanto, oito áreas.

Inspirada na experiência exitosa do Promed, Pró-Saúde I e Premus, a PUC-RS concebeu o Pró-Saúde II, com base em uma visão interdisciplinar e de construção coletiva, desde as etapas preliminares de formulação até o desenho do projeto em si e das estratégias de articulação intra e interinstitucional e com a comunidade, para a sua implementação. Para tanto, envolveram-se os cursos de graduação responsáveis pela formação dos profissionais das áreas de Farmácia, Nutrição, Fisioterapia, Medicina, Psicologia, Serviço Social e Odontologia, além do curso de Enfermagem, por meio do Pró-Saúde I. O princípio norteador da articulação, no âmbito da IES, foi contribuir para a formação integral dos estudantes, docentes e trabalhadores em saúde, com vistas à integração ensino-serviço, considerando a realidade social.

Essa articulação pressupôs um alinhamento entre as áreas de conhecimento quanto às atividades pedagógicas nos cenários das práticas da rede de serviços de atenção básica do município de Porto Alegre, assim como desencadeou o repensar dos currículos para adequá-los à formação para o SUS.

Tal processo se articulou com o Pró-Saúde I e com a Comissão Gestora Local preexistente, que se ampliou com a inclusão de novos atores nesse grupo. Para operacionalização da proposta, foi desenhado um conjunto de 15 projetos distribuídos nos três eixos do Pró-Saúde concebidos com enfoque interdisciplinar, constituindo uma proposta unificada. As articulações entre as áreas, bem como suas especificidades, foram expressas nas atividades previstas em cada um dos projetos. 
Em 2009, a primeira edição do PET-Saúde (2009-2010) foi aprovada, e a Universidade foi contemplada com quatro grupos envolvendo trabalhadores, docentes e alunos dos cursos de Enfermagem, Farmácia, Medicina, Nutrição, Odontologia, Psicologia e Serviço Social. Nesse período, foi realizado o Diagnóstico de Saúde do Distrito Nordeste de Porto Alegre, atendendo à solicitação da Secretaria Municipal de Saúde (SMS). Os resultados serão publicados em livro com apoio do Pró-Saúde I e II.

Na segunda edição do PET-Saúde/Saúde da Família (2010-2011), foram aprovados cinco grupos. Da mesma forma que na primeira edição, tutores, preceptores e acadêmicos atuam de forma multiprofissional e interdisciplinar. Esse novo projeto busca o desenvolvimento e a ampliação das atividades acadêmicas nos serviços de saúde, desenvolvendo intervenções com base nos problemas prioritários identificados na primeira fase da pesquisa - Diagnóstico de Saúde do Distrito Nordeste.

Adicionalmente, os alunos do PET-Saúde atuam em atividades específicas de intervenção nos serviços de atenção básica, desenvolvendo atividades assistenciais de sua área profissional, vinculadas ou não às disciplinas acadêmicas. Apesar de se vincularem às atividades da área profissional, é incentivada a integração ao trabalho de equipe, com apoio dos preceptores. Em relação à factibilidade e sustentabilidade, os bolsistas do PET-Saúde realizam atividades integradas com os residentes do Programa de Residência Multiprofissional em Saúde da Família, bem como com os bolsistas do Pró-Saúde, nos campos de prática.

Recentemente, a Universidade foi contemplada com um grupo do PET-Saúde/Vigilância em Saúde, cujo projeto será desenvolvido em parceria com a Equipe de Vigilância de Eventos Vitais, Doenças e Agravos Não Transmissíveis da Coordenadoria Geral de Vigilância em Saúde (GCVS) da SMS, e inclui os cursos de Fisioterapia e Educação Física, além dos demais já envolvidos no PET-Saúde/Saúde da Família. Esse trabalho permitirá a qualificação das ações de vigilância de agravos não transmissíveis junto à rede de saúde do município de Porto Alegre, contribuindo para alcançar a meta pactuada para a implementação de um sistema de vigilância da prevenção de câncer de colo de útero para mulheres de 25 a 59 anos de idade moradoras do município.

O Instituto Nacional do Câncer (Inca) estima que 500.000 novos casos de câncer do colo útero ocorram a cada ano no mundo, sendo responsável pelo óbito de, aproximadamente, 230 mil mulheres por ano. Sua incidência é cerca de duas vezes maior em países menos desenvolvidos em comparação com os mais desenvolvidos. A incidência por câncer do colo do útero torna-se perceptível na faixa etária de 20 a 29 anos, e o risco aumenta rapidamente até atingir seu pico na faixa etária de 45 a 49 anos $^{7}$. Entende-se que estratégias de investigação e de intervenção possibilitarão a elevação das condições de saúde da população de Porto Alegre, bem como a reorientação do processo educativo e a formação profissional na área da saúde.

Compõe ainda esse processo de articulação interna, no âmbito da PUC-RS, a busca constante de sinergia e integração de esforços, advindos do desenvolvimento de todos os projetos específicos que convergem ao mesmo propósito: integração ensino-serviço. Muitas das atividades planejadas por um dos projetos são compartilhadas com os demais programas, potencializando-se os investimentos em formação de recursos humanos, a integração ensino e serviço e o fortalecimento da qualidade dos serviços prestados à população, na perspectiva da ampliação da cidadania dos usuários.

Adicionalmente, os recursos dos projetos Pró-Saúde viabilizaram diversas melhorias nos serviços, ampliando a possibilidade de realização de atividades de ensino dos alunos de todas as graduações na área da saúde. Essas estratégias, somadas ao apoio das Pró-Reitorias de Graduação, Extensão, Pesquisa e Pós-Graduação, têm garantido a sustentabilidade e continuidade das atividades na rede de atenção básica. Os cursos estabeleceram estratégias de inserção dos alunos nos cenários de práticas nos serviços de atenção básica, da rede municipal de saúde de Porto Alegre, do Distrito Leste/Nordeste. É Importante salientar a contribuição da Gerência Distrital nesse processo, estabelecendo demandas prioritárias e criando condições objetivas para alocação dos alunos nos campos de estágio.

Até o presente momento, entre as disciplinas dos cursos da área da saúde envolvidos nos programas, 28 têm como cenário de prática a atenção básica, envolvendo, por semestre, cerca de 860 alunos dos cursos da graduação, 62 profissionais de saúde, 51 docentes, 13 tutores e 19 residentes, todos buscando trabalhar as ações propostas de forma integrada, ampliando, assim, uma nova concepção de trabalho multidisciplinar em saúde.

Nesse sentido, todos os autores envolvidos têm buscado contribuir para a melhoria da assistência à saúde da população, mediante capacitação dos profissionais, redes de aprendizado que envolva a academia e os serviços ${ }^{8}$ :

Além disso, salienta-se que a Universidade acolheu a proposta no âmbito de suas estratégias de gestão, criando uma Coordenadoria de Integração Ensino-Serviço na Área da Saúde, facilitando a gestão coordenada de processos administrativos e acadêmicos internos e de suas interfaces externas. 


\section{DESCOBERTAS E AVANÇOS}

Constata-se que as atividades realizadas têm fortalecido os conhecimentos norteadores das políticas de saúde e educação permanente em saúde, favorecendo o aprofundamento da integração ensino-serviço. A ampliação de referenciais foi um aspecto determinante, enfocando a atenção básica e a ampliação de práticas de ensino nos serviços na Gerência Distrital Leste/Nordeste. Essa estratégia tem propiciado mudanças na orientação teórica e ampliação da produção de conhecimento voltado às necessidades do SUS.

Dentre as descobertas e avanços, destaca-se o fortalecimento de ações conjuntas entre os cursos da área da saúde da PUC-RS, a partir de projetos como Premus, Pró-Saúde I e II e PET-Saúde. Tais projetos têm sido estratégicos para a reorientação da formação dos profissionais no âmbito da graduação e sua articulação com a pós-graduação, a fim de atender às necessidades de formação de recursos humanos para o SUS. Além disso, provocaram a ampliação da carga horária de estágios obrigatórios em serviços de saúde na atenção básica nos cursos da saúde, bem como a ampliação da integração ensino-assistência nas atividades de ensino/ pesquisa/extensão.

Outra consequência importante desses projetos foi o fortalecimento de mecanismos de cooperação entre os gestores municipais, os cursos da área da saúde e o Conselho Municipal de Saúde (CMS), os quais possuem representantes na Comissão Gestora Local. A integração entre SMS, CMS e a PUC-RS tem-se fortalecido e resultado em um aprofundamento da integração ensino-serviço, bem como na aquisição e troca de conhecimentos entre trabalhadores, docentes e discentes, estimulando a inovação.

É entendimento da Equipe de Desenvolvimento/CGADSS da SMS de Porto Alegre, como área responsável pelas ações de educação permanente em saúde, que a Comissão Gestora Local desses projetos constitui-se um espaço importante de reflexão, discussão, planejamento e avaliação das ações realizadas.

Deve ser destacada a maior satisfação dos usuários com os serviços prestados nos locais em que a Universidade está presente, o que conduz a um aumento e aprofundamento de ações e atividades de ensino e assistência no Distrito Leste/ Nordeste e à integração dos alunos e docentes em equipes multidisciplinares. Consequentemente, houve ampliação da assistência, principalmente na educação e promoção da saúde (em grupos, escolas, consultas e orientações), e foram implantados diversos programas com a participação efetiva de todos os atores envolvidos, o que qualifica o processo, pois todos os segmentos estão presentes.
Conforme o Conselho Municipal de Saúde: "as ações em saúde, ocorridas em decorrência da execução dos projetos do Pró-Saúde e do PET-Saúde, na área de abrangência da Gerência Distrital Leste/Nordeste e com a participação da PUC-RS, devem ser consideradas um acréscimo à rede de saúde do município como um todo".

É importante ressaltar que devem ser consideradas as ações em saúde, não somente a participação de estudantes e docentes, mas também a aquisição de bens patrimoniais, material de expediente e reformas. Foi possível a melhoria das instalações dos serviços, aquisição de equipamentos e materiais com apoio do Pró-Saúde. Portanto, salienta-se que tais investimentos contribuíram para que as equipes de saúde, estudantes e docentes, dentro desses locais de trabalho, pudessem desempenhar de forma mais satisfatória seus processos de trabalho.

Em relação a mudanças no ensino propriamente dito, esses projetos propiciam a realização de um maior número de capacitações e discussões entre docentes e trabalhadores, tendo em vista a metodologia de problematização na prática docente-assistencial. Adicionalmente, torna-se evidente uma maior participação dos profissionais do serviço no planejamento das estratégias pedagógicas nos cenários de prática. É importante destacar que o planejamento das ações em educação permanente em saúde desenvolvidas por meio do Pró-Saúde e PET-Saúde tem sido realizado em conjunto, considerando as necessidades e a realidade dos serviços e dos trabalhadores, articulado com as políticas setoriais da Secretaria Municipal de Saúde.

Entende-se que em função de várias ações colocadas em prática, desde oficinas até os citados benefícios de ordem material e patrimonial, houve uma desacomodação na cultura estabelecida, ou seja, houve uma provocação positiva no ânimo e no comportamento dos trabalhadores. Isso, certamente, já repercute nas suas relações com os usuários e com os estudantes e docentes da instituição.

\section{POTENCIALIDADES ENCONTRADAS}

Durante a primeira edição do PET-Saúde foi realizada a pesquisa Diagnóstico de Saúde do Distrito Nordeste. Essa atividade contribuiu de forma relevante para que os estudantes entendessem a importância das três dimensões da vida acadêmica: ensino, pesquisa e extensão. Os resultados da pesquisa foram utilizados para a definição de novas atividades e serão publicados em formato de livro.

Além disso, a inserção dos acadêmicos nos serviços tem demonstrado ser uma excelente oportunidade para conhecer o funcionamento integral de uma UBS e de uma ESF, a realidade 
do SUS e seus princípios, bem como os serviços prestados e as necessidades dos usuários, possibilitando maior integração da teoria com a prática, a multidisciplinaridade e a possibilidade de compartilhar saberes. Desse modo, deve ser estimulada continuamente a atitude de querer aprender e a pró-atividade em busca da autonomia, evidenciada por muitos dos alunos que propõem e desenvolvem estratégias e ações de inserção profissional no cenário do projeto.

Entre as diversas potencialidades encontradas, devem ser destacadas a boa articulação entre gestores, docentes, trabalhadores e acadêmicos, a presença de preceptores e tutores motivados e as diversas capacitações realizadas pela PUC-RS, integrando os programas Pró-Saúde I e II, PET-Saúde e Premus. Pode ser observada uma integração crescente entre os cursos da área da saúde da PUC-RS, o que tem motivado os alunos ao trabalho em equipe multiprofissional. Comparando com a integração existente antes desses projetos, pode-se dizer que houve um crescimento exponencial nesse âmbito, pois em algumas áreas os integrantes praticamente não dialogavam. Porém, ainda há espaço para uma maior integração, o que vem acontecendo com as novas ações propostas e o amadurecimento dos atores envolvidos nos processos.

É evidente que o aumento e o aprofundamento de ações e atividades na Gerência Distrital Leste/Nordeste proporcionaram a inserção progressiva de docentes e discentes no contexto do SUS, muitos dos quais não desenvolviam atividades nesse âmbito antes do surgimento dos programas. É possível observar a integração de diversas áreas do conhecimento no contexto da atenção primária à saúde. Muitas atividades integradoras têm sido sugeridas pelos acadêmicos, que, ao vivenciar as novas modalidades, motivam-se a fazer a integração dos conhecimentos e acabam provocando os docentes a participar dessa nova realidade, potencializando a interdisciplinaridade. Como consequência, observa-se a crescente identificação dos acadêmicos e docentes com a atenção básica, os quais demonstram um forte interesse de inserção profissional em serviços públicos, atendendo aos princípios e diretrizes do SUS.

Como importante potencial deve ser destacada a necessidade de ampliar a divulgação dos resultados de trabalhos e pesquisas realizados pelos alunos para as equipes, comunidade e sociedade de um modo geral.

\section{DIFICULDADES E PONTOS CRÍTICOS}

Naturalmente, algumas dificuldades têm sido encontradas. Em relação aos serviços, destaca-se: o fato de algumas equipes de saúde apresentarem demandas de vagas para bolsistas acima da sua capacidade; a dificuldade de inserção de docentes e estudantes em locais de difícil acesso e com espaço físico reduzido: e a dificuldade para inserir estudantes de algumas áreas da saúde nas UBSs e ESFs por falta de profissionais dessas áreas e/ou inexistência de atividades multidisciplinares.

Ressalta-se ainda, como desafio, a necessidade de aumentar o número de intervenções realizadas conjuntamente por acadêmicos e profissionais de duas ou mais áreas. Esse fato decorre, dentre outros fatores, da rotina dos serviços, de horários de aulas não coincidentes entre os diversos cursos e da falta de cultura de alguns trabalhadores e docentes para desenvolver atividades multiprofissionais, engendradas por uma concepção interdisciplinar. Percebe-se que a grande demanda de atividades de rotina nos serviços de saúde dificulta a participação dos trabalhadores em atividades com os acadêmicos, bem como em reuniões de planejamento e de capacitação.

Em relação à integração dos acadêmicos e docentes dos diversos cursos de graduação da área da saúde, ela é dificultada pelo fato de os cursos possuírem níveis diferentes de inserção em atividades na atenção básica, bem como pela inexistência de disciplinas comuns para as graduações e pelo fato de os horários de aulas serem muito diversos, tornando difícil a realização de trabalhos multidisciplinares. A criação de mais oportunidades de encontro entre os estudantes de graduação das áreas envolvidas possibilitaria uma vivência multidisciplinar e interdisciplinar entre eles, potencializando a integralidade como princípio da formação profissional.

Como ponto crítico, torna-se evidente a necessidade de se trabalhar com a resistência, por parte de alguns trabalhadores, em viabilizar a integração ensino-assistência ou entender a inserção de uma equipe estendida nas Estratégias Saúde da Família. Observa-se que alguns trabalhadores apresentam dificuldades em participar das ações desses programas, talvez pela falta de conhecimento ou clareza quanto aos objetivos dos projetos.

Apesar de diversas melhorias nos serviços terem sido implantadas com apoio dos projetos, é importante destacar que a adequação da infraestrutura da rede de atenção à saúde, quanto à melhoria dos espaços físicos, equipamentos e materiais disponíveis, deve ser contínua. Outros aspectos que têm dificultado a realização das atividades são a precariedade da rede de serviços em saúde mental e a inexistência de Núcleos de Apoio à Saúde da Família (Nasf) no município de Porto Alegre.

De acordo com o Conselho Municipal de Saúde, para os usuários, o grande desafio do Pró-Saúde e do PET-Saúde é medir, por meio das avaliações e de forma qualificada, as repercussões de um acolhimento/atendimento de qualidade. Com a entrada em cena de docentes e estudantes atuando junto com os trabalhadores, há certamente uma oxigenação, 
uma motivação e, com isso, entende-se que as perspectivas são de alteração na cultura estabelecida, devendo essas serem avaliadas.

\section{EXPERIÊNCIAS BEM-SUCEDIDAS E SUSTENTABILIDADE}

Entre as experiências bem-sucedidas, destaca-se o acolhimento aos novos alunos bolsistas com a presença de representantes do Conselho Municipal de Saúde, tutores, preceptores, Gerência Distrital e Equipe de Desenvolvimento da SMS. Nessa oportunidade, foram apresentadas informações a respeito dos programas, da estrutura e políticas setoriais da SMS, Gerência Distrital Leste/Nordeste e sobre o Controle Social, bem como as expectativas em relação aos projetos.

Vale ressaltar que a participação dos alunos dos diferentes programas nos serviços de saúde tem servido de estímulo para a qualificação e aprimoramento técnico dos trabalhadores, trazendo repercussões positivas ao serviço.

Além disso, a relação entre os processos de ensino e a prestação de serviços tem proporcionado uma formação diferenciada aos acadêmicos, que passam a vivenciar os desafios de materialização dos princípios do SUS na atenção básica em saúde, estimulando a formação de recursos humanos para atuação na rede de serviços do sistema.

Em relação à sustentabilidade, o Pró-Saúde tem possibilitado um aporte de investimentos para a melhoria dos serviços, especialmente em equipamentos audiovisuais e de Informática (computadores), e para a capacitação de profissionais, visando à qualificação da integração ensino-serviço.

A integração de atividades da graduação (PET-Saúde e Pró-Saúde) com a pós-graduação, por meio da residência, possibilita a potencialização dos recursos financiados, com vistas à integração ensino-serviço e reorientação na formação profissional. Como exemplo, cita-se a realização da I Mostra PET-Saúde, Premus, Pró-Saúde, com apresentação de 57 trabalhos e 224 participantes, entre bolsistas PET-Saúde e Pró-Saúde, residentes Premus, docentes, trabalhadores, representantes da SMS, representantes do Controle Social, coordenadores de curso e diretores de Unidades Acadêmicas.

Ressalta-se também a ampliação do número de trabalhos de conclusão de curso articulados com os programas, a inserção de trabalhadores em projetos de pesquisas desenvolvidos por professores e alunos e as capacitações, contemplando todos os segmentos: docentes, estudantes, tutores, preceptores, residentes, trabalhadores de diversas áreas e das demais Gerências Distritais da SMS, gestores e Controle Social. Essas ações têm oportunizado um espaço de encontro e problematização que contribui para reflexão, discussão e troca de experiências.
$\mathrm{O}$ reconhecimento do trabalho coletivo e interinstitucional realizado culminou na premiação da PUC-RS, pelo Conselho Municipal de Saúde de Porto Alegre, no dia 20 de maio de 2010, com o Prêmio Destaque em Saúde, que foi concedido a personalidades e instituições que se destacaram nas ações de saúde no município em diversas modalidades. A PUC-RS recebeu a distinção na área de ensino, principalmente pela contribuição às ações realizadas nos programas Pró-Saúde, Pet-Saúde e Premus.

\section{CONSIDERAÇÕES FINAIS}

Espera-se, a partir das diversas ações realizadas com apoio do PET-Saúde, PRÓ-Saúde e Premus, promover maior efetividade nas atividades de promoção de saúde, bem como integração da equipe de saúde e qualificação do ensino. Nesse sentido, destaca-se a necessidade de se trabalhar continuamente as diferentes concepções de saúde e de modelo assistencial com os atores envolvidos: docentes, estudantes, trabalhadores, gestores e usuários. Também deve ser ampliado o número de docentes integrados às equipes de saúde, a fim de relacionar conteúdos específicos com a atenção básica, bem como ser ampliada a carga horária de atividades de graduação relacionadas à atenção básica.

É importante que os acadêmicos participem de reuniões de equipe, do Conselho Municipal de Saúde, distritais e locais, a fim de compreender ainda mais a realidade e propor ações que atendam às necessidades da comunidade. A vivência na gestão, planejamento e formação, sempre privilegiando o trabalho em equipe, deve ser permanentemente incentivada, visando à integralidade na formação dos profissionais da saúde.

Para qualificar o processo de avaliação das ações e capacitações dos diversos programas, devem ser elaborados indicadores e instrumentos de avaliação, identificando, também, o impacto junto aos usuários.

A fim de dar continuidade aos avanços proporcionados pelos projetos, é necessário ser ampliado o debate sobre o projeto político-pedagógico da formação em saúde, incentivando o compromisso do ensino com o SUS. Além disso, deve ser estimulada a discussão entre as disciplinas que abordam Saúde Coletiva e o SUS nos diversos cursos da saúde, com o objetivo de planejar os conteúdos de forma integrada. Para tanto, uma das estratégias propostas é integrar os estudantes dos cursos da saúde em uma mesma disciplina de Saúde Coletiva durante sua formação.

Outra importante perspectiva é estimular o debate e práticas relativas à educação permanente em saúde e a interação entre educação e processos de trabalho, a partir da realidade vivenciada, levando em consideração os conhecimentos e as 
experiências de todos. Além disso, deve ser incentivada a reflexão e a discussão sobre o papel do preceptor e dos demais trabalhadores na formação dos estudantes e a responsabilidade dos docentes com os serviços de saúde.

\section{REFERÊNCIAS}

1. Albuquerque VS. A integração ensino-serviço no contexto dos processos de mudança na formação superior dos profissionais da saúde. Rev. bras. educ. med. [online]. 2008, vol.32, n.3 [cited 2009-12-17], pp. 356-362. Available from: <http: //www.scielo.br/scielo.php?script=sci_ arttext\&pid=S0100-

2. Moretto A. Saúde como direito: desafio para o ensino em saúde. In: PUCRS. Programa de Incentivo a Mudanças Curriculares nos Cursos de Medicina. Diagnóstico de saúde do distrito leste de Porto Alegre: PROMED. Porto Alegre: EDIPUCRS, 2005, p. 203-244.

3. Mendes JMR. Condições de vida, qualidade dos serviços de saúde e rede social do distrito leste. In: PUCRS. Programa de Incentivo a Mudanças Curriculares nos Cursos de Medicina. Diagnóstico de saúde do distrito leste de Porto Alegre: PROMED. Porto Alegre: EDIPUCRS, 2005, p. 81 202.

4. Pereira MG. Epidemiologia: teoria e prática. Rio de Janeiro: Guanabara Koogan, 1997.

5. Brasil. Ministério da Saúde. Secretaria de Vigilância em Saúde. Departamento de Análise de Situação de Saúde. Saúde Brasil 2004: uma análise da situação de saúde. Brasília: Ministério da Saúde, 2004.
6. Ferreira MLSM, Cotta RMM, Oliveira MS. Construção coletiva de experiências inovadoras no processo ensino-aprendizagem na formação de profissionais da saúde. Rev. bras. educ. med. [online]. 2009, vol.33, n.2, pp. 240-246. ISSN 0100-5502.

7. Brasil. Ministério da Saúde. Instituto Nacional de Câncer. Câncer de colo de útero. Disponível em: http: //www2. inca.gov.br/wps/wcm/connect/tiposdecancer/site/ home/colo_utero/definicao Acesso em: 15/06/2010.

8. Campos FE, Brenelli SL, Lobo LC, Haddad AE. O SUS como escola: a responsabilidade social com a atenção à saúde da população e com a aprendizagem dos futuros profissionais de saúde. Rev. bras. educ. med. [online]. 2009, vol.33, n.4, pp. 513-514. ISSN 0100-5502.

\section{CONTRIBUIÇÃO DOS AUTORES}

Todos os autores participaram na eleboração do manuscrito, desde a sua comcepção até a finalização do texto.

\section{CONFLITO DE INTERESSES}

Declarou não haver

\section{ENDEREÇO PARA CORRESPONDÊNCIA}

Adolfo Pizzinato

Av. Ipiranga, 6681, Prédio 11, 8º andar

Porto Alegre

CEP. 90619-900 - RS

E-mail: adolfo.pizzinato@pucrs.br. 